\title{
"Minimally invasive" regional anesthesia and the expanding use of interfascial plane blocks: the need for more systematic evaluation
}

\author{
Rakesh V. Sondekoppam, MD • Ban C. H. Tsui, MD
}

Received: 15 April 2019/Revised: 15 April 2019/Accepted: 15 April 2019/Published online: 21 May 2019

(C) Canadian Anesthesiologists' Society 2019

Just as "minimally invasive surgery" has seen significant advances in recent years, so has the field of regional anesthesia. Over the last decade, regional anesthesia has seen a shift towards decreasing utilization of central neuraxial blocks in both adult ${ }^{1}$ and pediatric patients ${ }^{2}$ that has been matched with a growing popularity in lesser invasive approaches to peripheral nerve blocks, most recently with the increased use of interfascial blocks. While this is likely due to a variety of reasons, the move towards "minimally invasive blocks" parallels similar endeavours in the surgical specialties.

Minimally invasive surgical techniques have shown to result in faster recovery, better patient comfort, and reduced hospital length of stay, to the point where traditional neuraxial techniques may not provide significant comparable analgesic benefits. ${ }^{3}$ Regional analgesic regimens such as thoracic epidural analgesia (TEA), paravertebral blocks, and intrathecal opioids are associated with the risk of major complications such as neuraxial hematoma, pneumothorax, and respiratory depression. In addition, despite subjecting the patients to the risk of these major complications, these traditional analgesic techniques also have a significant incidence of failure. There are also hidden patient and economic burdens of maintaining and troubleshooting these neuraxial techniques. The increasing popularity of oral

R. V. Sondekoppam, MD

Department of Anesthesiology, University of Alberta,

Edmonton, AB, Canada

B. C. H. Tsui, MD ( $\square)$

Department of Anesthesiology, Perioperative and Pain Medicine, Stanford University School of Medicine, 300 Pasteur Drive,

Stanford, CA, USA

e-mail: bantsui@stanford.edu anticoagulants and low molecular weight heparins have made monitoring a patient's coagulation status challenging, and when coupled with the higher cost of maintaining continuous catheters, additional investigations to rule out complications (such as pneumothorax or spinal epidural hematoma) may have played a role in these techniques falling out of favour. Hence, there is a paradigm shift in anesthesia towards more minimally invasive approaches to improve the risk/benefit profile of analgesic techniques.

Plato famously wrote that "Necessity is the mother of invention" and this aptly describes the current trends in ultrasound-guided minimally invasive block approaches. Indeed, an expanding number of interfascial blocks are being described to target various fascial planes instead of defined neural bundles targeted by traditional regional anesthesia. ${ }^{4}$ Since most interfascial plane blocks address targets outside the central neuraxis, and at a distance from other major nerves, they are thought to decrease the incidence of major complications and side effects such as hypotension, or avoid the risk of direct trauma to neurological structures.

In the current issue of the Journal, Aponte et al. ${ }^{5}$ present an anatomical study of the injectate pattern of spread in cadavers for the relatively newly described interfascial erector spinae plane (ESP) block. ${ }^{6}$ The ESP block is one of the paravertebral block alternatives that has been described in recent times. Others include the retrolaminar block and the midpoint transverse process to pleura block; all of these alternate blocks are broadly considered paravertebral-byproxy. ${ }^{7}$ Aponte et al. in their present study found no paravertebral spread of radiocontrast dye in any of the ESP injections, with the injectate mainly being confined to the region posterior to the transverse process, the lamina, and along the interfascial plane beneath the erector spinae muscle. 
Current evidence for the spread of an ESP injectate into the paravertebral space (PVS) is somewhat conflicting, with studies both supporting ${ }^{8-10}$ and refuting ${ }^{11}$ the spread of the injectate through the costo-transverse ligament into the PVS following an ESP block. There are several methodological issues common to cadaveric studies that might be responsible for these discrepancies. Cadaveric studies, and other forms of basic science research, are more commonly the starting points of evaluating newer techniques. Nevertheless, obtaining evidence of any block's utility is an arduous task and it may take a long time before conclusive recommendations can be made. For example, the initial description of the transversus abdominis plane (TAP) block dates back to $2001,{ }^{12}$ and subsequently, a variety of approaches have been described $^{13}$ with evidence both for ${ }^{14}$ and against ${ }^{15}$ its analgesic efficacy. There is still a need for large-scale multicentre randomized clinical trial to evaluate this technique. Nevertheless, even the synthesis of existing evidence concerning its utility in comparison with "gold standard" techniques such as TEA has taken significant time to acquire since its first description. Although robust and direct comparative trials of TEA and TAP are still required to make definitive conclusions, the recent metaanalysis of randomized clinical trials has suggested that TAP blocks provide acceptable analgesia (albeit in the background of multimodal analgesia) and have a better side-effect profile with a lower incidence of hypotension and a reduced length of hospital stay compared with TEA. ${ }^{16}$ Nevertheless, the take home message is that such delays in evidence generation need to be minimized in order to offer evidence-based analgesic options.

Regional anesthesia is currently facing a scenario comparable to that of procedural interventions in surgical specialties where the evidence for efficacy and regulatory oversight are often disconnected. ${ }^{17}$ Hence, better approaches to evidence generation need to be employed, especially with the current pace in the description of newer techniques of regional analgesic techniques; perhaps nonanalgesic outcomes also need to be factored into the evaluation of their utility. In this editorial, we present the reasoning for the need to standardize the conduct of cadaveric studies, and also briefly discuss approaches to better assimilate clinical evidence from the newer regional anesthesia procedures.

\section{Anatomical studies on interfascial plane blocks: extrapolate with caution}

Cadaveric studies usually have small sample sizes and accordingly, a high risk for bias. Moreover, the anatomical variability of the cadavers and physiologic difference between living and dead tissues points to the use of cadaveric studies more for hypothesis generation than uncovering unequivocal mechanisms of action. Given these caveats, cadaveric studies are considered the lowest valid form of evidence for evaluating therapeutic interventions, ${ }^{18}$ but nevertheless, provide some valuable insight and data to improve clinical practice.

Nevertheless, cadaveric models need to be standardized for better evaluation of injectate dispersion and to compare and contrast among the various studies. No cadaveric model can fully mimic a live patient as it lacks key factors important for injectate dispersion, including blood circulation and muscle contraction. In addition, post mortem changes to the tissue architecture can further impact the injectate spread. ${ }^{19}$ While fresh cadavers are considered more realistic as any post mortem tissue changes are minimal, their use may be more relevant for surgical simulations than studies in regional anesthesia. Cadaveric models for regional anesthesia have mostly been developed with the aim of improving the visualization of anatomical structures and the needle. ${ }^{20}$ Among the many models, Thiel embalmed cadavers are known to provide the best physical and functional properties for peripheral nerve blocks $^{21,22}$ and perhaps should be validated to evaluate interfascial plane blocks.

There is also a need to determine the ideal injectate composition for evaluating injectate spread following regional analgesic techniques. Injectates used in the evaluation of cadaveric spread have been variable in composition and consistency, and include methylene blue $^{9-11}$ and latex, ${ }^{8}$ both of which have been used in ESP studies; more complex formulations have been used for other interfascial plane blocks. ${ }^{23,24}$ The varying viscosity of these solutions may impact the resultant spread as aqueous dyes may diffuse more easily through tissues compared with viscous dyes.

Independent of the injectate composition, the technique of performing these blocks needs to be standardized as the direction of the needle/bevel and the pressure generated during the injection could potentially impact fluid dynamics and thus the resultant dye spread. ${ }^{25}$ A detailed description of the injection technique is particularly important information to understand and to determine the resultant injectate spread; it needs to be factored in when interpreting the results of cadaveric studies. Consistent evaluation needs to take into account specific technical considerations such as whether the injection was using an in-plane $v s$ an out-of-plane approach, or a medial to lateral $v s$ a lateral to medial approaches. In the case of interfascial blocks such as ESP, an additional consideration such as a cephalad- or caudad-directed needle injection can also impact greatly on the spread of injectate. Furthermore, it is also important to know if the operator performing the block 
has had adequate experience with the technique when newer procedures are being evaluated, as experience may also impact the success of the block.

Despite the need to standardize certain steps in cadaveric studies of regional anesthesia, these studies may still not completely explain the mechanism of analgesia because regional anesthetic techniques may involve both loco-regional and systemic effects of local anesthetics. ${ }^{26,27}$ For loco-regional effects, the muscular and fascial tissue in the intended area for these blocks can actively contract, ${ }^{28}$ and whether or not such changes in musculoskeletal dynamics influence injectate spread cannot be determined from cadaveric studies. In addition, the clinical analgesic effect of local anesthetic from its systemic absorption obviously cannot be evaluated in a cadaveric study. This is particularly important as certain local anesthetics such as lidocaine have clearly exhibited powerful analgesic effects when given intravenously. ${ }^{29}$

Hence, anatomical and radiological studies are designed to explain the plausibility of a technique and do not account for live tissue characteristics, anatomical variability, performer learning curve, or observer bias. Despite the weaknesses of these anatomical studies, one key lesson from them is that there appears to be considerable variability in injectate spread. That said, while the mechanism of analgesia itself needs further exploration, it is also important to consider the utility of interfascial blocks in everyday practice. There is a need for generating timely quality clinical evidence for these techniques before newer techniques are offered as the standard of care.

\section{Lack of high-quality evidence with newer block: Is it worth waiting?}

Multicentre randomized-controlled trials (RCT) (along with meta-analyses of the same) are considered to provide the highest level evidence concerning the clinical efficacy of a drug, device, or technique. Nevertheless, highquality comparative studies of newer interfascial plane blocks largely do not exist. While a single centre study is often critiqued for a lack of scientific rigor and external validity, ${ }^{30}$ large-sample multicentre studies are not only cost intensive, but also take a long time to complete. Generating timely evidence is crucial as a delay in generating high-quality evidence regarding the safety or efficacy of a technique before adoption may deny deserving patients access to potentially superior techniques, while the widespread adoption of these techniques before the availability of definitive evidence runs contrary to the principles of beneficence. Also, and as pointed out by Wallis et al., ${ }^{17}$ once a procedure is perceived as a standard of care, there may be difficulty and resistance in removing such techniques from practice despite the availability of evidence against a technique. Data collection and analysis from patient registries, big data analyses, and large-scale observational studies may be

Table Evaluating analgesic efficacy of a new regional anesthesia technique

\begin{tabular}{|c|c|c|c|}
\hline Type of study & Study characteristics to consider & Keep in mind & Level of evidence \\
\hline Cadaveric studies & $\begin{array}{l}\text { - Is the cadaveric model life-like? } \\
\text { - Is the injectate influencing the spread? } \\
\text { - Anatomical variations? } \\
\text { - Technical considerations: pressure of } \\
\text { injection, bevel or needle orientation, } \\
\text { performer learning curve/experience }\end{array}$ & $\begin{array}{l}\text { - Is it confirmed or refuted by other studies? } \\
\text { - Performer and observer bias }\end{array}$ & - Plausibility studies \\
\hline Case series/reports & $\begin{array}{l}\text { - Indications for the block may vary } \\
\text { - May denote clinical utility and help in } \\
\text { generating study hypothesis }\end{array}$ & $\begin{array}{l}\text { - Publication bias (negative studies may not } \\
\text { be published because of the fear of a lack of } \\
\text { experience with the new technique) }\end{array}$ & $\begin{array}{l}\text { - Anecdotal evidence (useful } \\
\text { for early clinical experience } \\
\text { and adverse event reporting) }\end{array}$ \\
\hline Single-centre RCT & $\begin{array}{l}\text { - Internal validity } \\
\text { - External validity }\end{array}$ & $\begin{array}{l}\text { - It is rare to refute or accept findings of a } \\
\text { single RCT for practice consideration }\end{array}$ & $\begin{array}{l}\text { - Hypothesis generating studies } \\
\text { - Needs replicative studies }\end{array}$ \\
\hline Multicentric RCT & $\begin{array}{l}\text { - External validity a concern if inclusion } \\
\text { criteria is too stringent }\end{array}$ & Usually time and cost intensive & $\begin{array}{l}\text { - Definitive evidence (but may } \\
\text { not be available on time) }\end{array}$ \\
\hline $\begin{array}{l}\text { Non-randomized } \\
\text { trials } \\
\text { (observational } \\
\text { studies, registry } \\
\text { trials, database } \\
\text { studies) }\end{array}$ & $\begin{array}{l}\text { - May lack internal validity } \\
\text { - May be a more pragmatic approach }\end{array}$ & $\begin{array}{l}\text { - May need adaptive designs to emulate a } \\
\text { clinical trial }\end{array}$ & $\begin{array}{l}\text { - May be the best form of } \\
\text { evidence in the lack of } \\
\text { definitive evidence }\end{array}$ \\
\hline
\end{tabular}

$\mathrm{RCT}=$ randomized controlled trial 
needed to evaluate the utility and safety of emerging regional anesthesia techniques.

\section{What to consider when evaluating newer analgesic techniques}

When the analgesic efficacy is being considered, physicians need to carefully consider the study design and the caveats associated with each of them (Table). A systematic stepwise evaluation has been developed (Table) to mimic the developmental phases of a clinical trial in medicine. ${ }^{31}$ Using this approach, after preclinical trials (which in regional anesthesia are equivalent to cadaveric studies) are done, the drug/technique of interest undergoes various stages of evaluation. The first stages are exploratory studies (phase 0: case reports/series reporting on the preliminary experience, optimal dosing, adverse effects or complications of a newer block), then clinical effectiveness is examined in comparative studies, first in limited populations (phase 2: comparable to single-centre RCT) and then in larger populations (phase 3: large-scale multicentric RCT or well-designed non-randomized trials). The newer blocks also need to be evaluated from time to time using an established health technology assessment (HTA), which is a systematic multidisciplinary evaluation of evidence with respect to the safety, efficacy, economic, and social impact of an existing or new medical device,

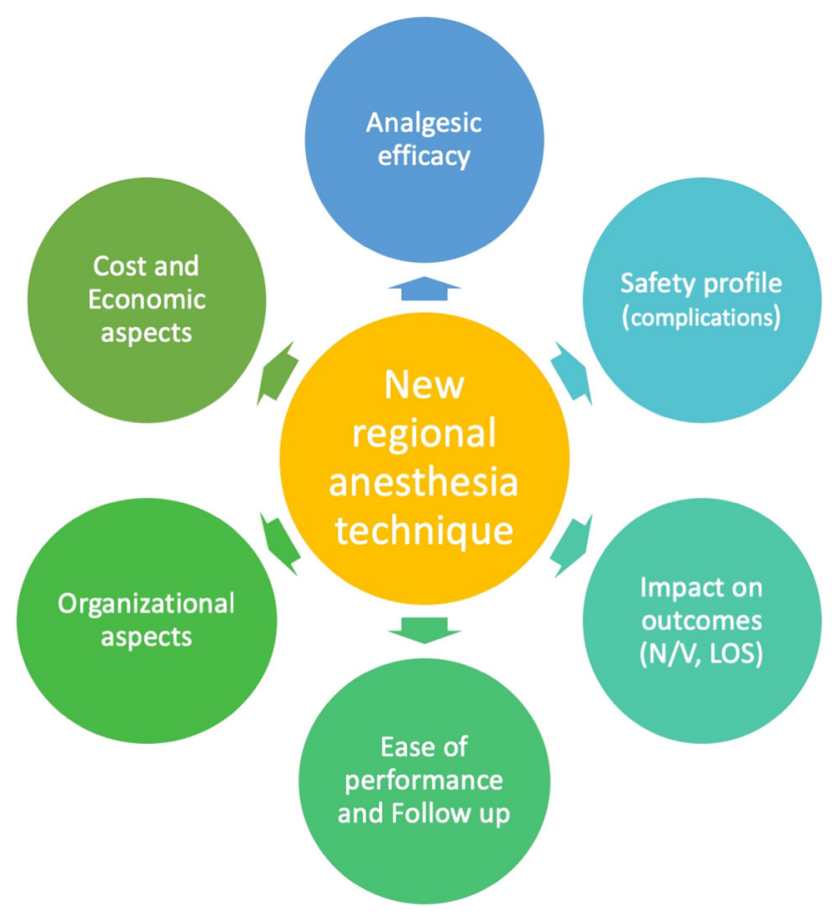

Figure Pragmatic scoring approachApproche de gradation pragmatique drug, or procedure to aid in the decision making. ${ }^{32}$ There is a growing realization that non-analgesic outcomes are equally important for postoperative patient comfort and a recent systematic review has attempted to standardize these endpoints, which include postoperative nausea and vomiting, quality-of-recovery scales, gastrointestinal recovery, mobilization, and sleep quality. ${ }^{33}$ Perhaps subjecting existing and newer regional anesthetic procedures to regular HTA at regular intervals could be considered equivalent to a phase 4 clinical trial where system factors and non-analgesic aspects of patient comfort are also considered.

Individual practitioners and institutions need to consider the best level of evidence available for each technique. It is our opinion that, at the very least, single-centre RCTs are conducted before the technique is adopted and that the technique is evaluated when a higher level of evidence becomes available at regular intervals. The impact of a newer analgesic technique in the long run should consider analgesic efficacy and other factors such as side effects (nausea/vomiting), non-pain outcomes (time to ambulation or length of hospital stay), ease of performance (especially by non-regional anesthesiologists), safety profile, ease of maintenance/follow-up, cost-effectiveness, and other organizational aspects (Figure). ${ }^{34}$ The use of a pragmatic scoring system, as proposed by Mudumbai et al. ${ }^{34}$ is a step in the right direction as it considers other aspects of care such as improving outcomes, increasing access to care, decreasing disparities, and improving efficiency.

While we appreciate that physicians who are eager to offer newer procedures have the patient's best interests in mind, the efficacy and safety data of these blocks need to be constantly updated, thus balancing the risks and benefits in our everyday practice.

\section{De la nécessité d'une évaluation plus rigoureuse de l'anesthésie régionale « minimalement invasive » et de l'utilisation croissante des blocs des plans interfasciaux}

Le domaine de l'anesthésie régionale a été témoin d'importants progrès au cours des dernières années, tout comme la « chirurgie minimalement invasive ». Au cours de la dernière décennie, nous avons assisté à une diminution de l'utilisation, en anesthésie régionale, des blocs neuraxiaux centraux (tant chez les patients adultes ${ }^{1}$ qu'en pédiatrie. ${ }^{2} \mathrm{La}$ diminution de l'intérêt pour ces approches est venue de paire avec la popularité croissante 
d'approches moins invasives des blocs nerveux périphériques, dont la plus récente illustration est l'utilisation croissante des blocs de plans interfasciaux. Bien que plusieurs raisons expliquent probablement ce phénomène, cet engouement pour les «blocs minimalement invasifs » peut être mis en parallèle avec des tendances semblables observées dans les spécialités chirurgicales.

Il a été démontré que les techniques chirurgicales minimalement invasives s'accompagnaient d'une récupération plus rapide, d'un meilleur confort pour le patient et d'une réduction de la durée de séjour hospitalier - à tel point que les techniques neuraxiales traditionnelles pourraient ne pas procurer de bienfaits analgésiques comparables. ${ }^{3}$ Les régimes d'analgésie régionale tels que l'analgésie péridurale thoracique (APT), les blocs paravertébraux et les opioïdes intrathécaux sont associés à un risque de complications majeures telles que l'hématome neuraxial, le pneumothorax, et la dépression respiratoire. En outre, en plus de soumettre les patients au risque de ces complications majeures, ces techniques analgésiques traditionnelles ont également une incidence significative d'échec. Qui plus est, il existe des fardeaux cachés et pour le patient, et d'un point de vue économique, lorsqu'on souhaite maintenir ces techniques neuraxiales et en régler les problématiques. La popularité croissante des anticoagulants oraux et des héparines à bas poids moléculaire a rendu le monitorage de la coagulation plus difficile, et si l'on ajoute à cela le coût plus élevé du maintien des cathéters continus, les examens supplémentaires nécessaires à exclure les complications (telles que le pneumothorax ou l'hématome épidural), ces facteurs pourraient avoir contribué à faire diminuer la popularité de ces techniques. Dès lors, on assiste à un virage en anesthésie vers des approches plus minimalement invasives afin d'améliorer le profil de risques et bienfaits des techniques analgésiques.

«La nécessité est mère de l'invention », disait Platon; cette phrase décrit de façon très juste les tendances actuelles dans les approches minimalement invasives de blocs échoguidés. En effet, un nombre croissant de blocs de plans interfasciaux sont décrits, lesquels ciblent divers plans fasciaux plutôt que des nerfs ciblés par l'anesthésie régionale traditionnelle. ${ }^{4}$ Étant donné que les cibles de la plupart des blocs de plan interfasciaux se trouvent à l'extérieur de l'axe neural central et relativement éloignées des autres nerfs majeurs, on pense qu'ils réduisent l'incidence de complications et d'effets secondaires majeurs tels que l'hypotension, ou évitent le risque de traumatisme direct aux structures neurologiques.

Dans ce numéro du Journal, Aponte et coll. présentent une étude anatomique sur la dispersion d'un produit injecté chez des cadavres lors de la réalisation d'un bloc relativement récent, soit le bloc du plan interfascial des muscles érecteurs du rachis (ESP). ${ }^{6}$ Le bloc ESP constitue l'une des alternatives les plus récemment décrites au bloc paravertébral. Les autres approches décrites comportent le bloc rétrolaminaire ainsi que le bloc du milieu de l'apophyse transverse et de la plèvre; ces autres blocs sont tous considérés comme étant des blocs paravertébraux par procuration. ${ }^{7}$ Dans leur étude publiée ici, Aponte et coll. n'ont observé aucune dispersion paravertébrale du produit de contraste dans toutes leurs injections ESP, le produit se confinant principalement à la région postérieure de l'apophyse transverse, de la lame vertébrale, et le long du plan interfascial sous les muscles érecteurs du rachis.

Les données probantes actuelles concernant la dispersion d'une injection ESP dans l'espace paravertébral (EPV) sont quelque peu problématiques, avec certaines études soutenant ${ }^{8-10}$ et d'autres réfutant ${ }^{11}$ la dispersion du produit injecté à travers le ligament costotransversaire dans l'EPV après un bloc ESP. Les études cadavériques s'accompagnent fréquemment de divers problèmes méthodologiques, lesquels pourraient expliquer ces disparités. Les études cadavériques, et d'autres formes de recherche en science fondamentale, sont fréquemment les points de départ utilisés pour évaluer les techniques plus récentes. L'obtention de données probantes concernant l'utilité d'un bloc est toutefois une tâche ardue, et il peut se passer un certain temps avant qu'une recommandation concluante ne puisse être émise. Par exemple, la première description d'un bloc du plan du muscle transverse de l'abdomen (TAP) remonte à 2001, ${ }^{12}$ par la suite, diverses approches ont été décrites, ${ }^{13}$ accompagnées de données probantes soutenant ${ }^{14}$ ou réfutant ${ }^{15}$ son efficacité analgésique. Cette technique n'a toujours pas été évaluée par une étude clinique randomisée multicentrique d'envergure. De plus, même la synthèse des données probantes existantes concernant l'utilité de ce bloc par rapport à des techniques considérées comme traitement de référence - telles que l'analgésie péridurale thoracique - a pris beaucoup de temps depuis sa première description. Bien que des études comparatives directes et rigoureuses entre l'analgésie péridurale thoracique et le bloc du plan du muscle transverse de l'abdomen soient encore nécessaires afin de parvenir à des conclusions définitives, une métaanalyse récente des études cliniques randomisées porte à croire que le bloc du plan du muscle transverse de l'abdomen procure une analgésie acceptable (si elle s'inscrit dans le cadre d'une analgésie multimodale) et présente un meilleur profil d'effets secondaires, avec une incidence plus faible d'hypotension et une durée réduite de séjour à l'hôpital, que l'analgésie péridurale thoracique. ${ }^{16}$ Ce qu'il faut retenir, c'est qu'il faut réduire au maximum les délais dans la génération des données probantes afin de 
pouvoir offrir des options analgésiques fondées sur des données probantes.

L'anesthésie régionale se trouve actuellement dans une situation comparable à celle des interventions procédurales dans les spécialités chirurgicales, où les données probantes d'efficacité et la supervision règlementaire sont complètement déconnectées. 17 Dès lors, il faudrait trouver des approches plus efficaces pour générer des données probantes, particulièrement étant donné la rapidité à laquelle de nouvelles techniques sont décrites en analgésie régionale; par exemple, les résultats non analgésiques devraient peut-être également être pris en compte dans l'évaluation de leur utilité. Dans cet éditorial, nous présentons notre réflexion, selon laquelle la réalisation d'études cadavériques devrait être standardisée, et nous présentons brièvement quelques approches qui nous permettraient de mieux intégrer les données probantes cliniques tirées des procédures d'anesthésie régionale plus récentes.

\section{Études anatomiques sur les blocs de plans interfasciaux : prudence lors de l'extrapolation}

Les études cadavériques ont en général des tailles d'échantillon réduites et sont, dès lors, à haut risque d'être victimes de biais. En outre, en raison de la variabilité anatomique des cadavres et des différences physiologiques entre les tissus vivants et morts, il faut garder à l'esprit que les études cadavériques devraient être utilisées pour générer des hypothèses plutôt que pour déterminer de façon concluante et sans équivoque un quelconque mécanisme d'action. Étant donné ces écueils, les études cadavériques sont considérées comme la forme la plus faible de données probantes pour l'évaluation des interventions thérapeutiques ${ }^{18}$ - ce qui ne les empêche pas de fournir des perspectives et des données précieuses pour améliorer la pratique clinique.

Les modèles cadavériques doivent toutefois être standardisés afin de pouvoir mieux évaluer la dispersion des produits injectés ainsi que de comparer et mettre en contraste les résultats tirés de diverses études. Aucun modèle cadavérique ne peut imiter parfaitement un patient en vie : certains facteurs clés pour évaluer la dispersion du produit injecté sont absents d'un tel modèle, notamment la circulation sanguine et les contractions musculaires. En outre, les changements post mortem de l'architecture tissulaire peuvent également avoir un impact sur la dispersion du produit injecté. ${ }^{19}$ Alors que les cadavres frais sont considérés comme des modèles plus réalistes, les changements tissulaires post mortem y étant minimaux, ce type de modèle serait plus utile et pertinent pour des simulations chirurgicales que pour des études en anesthésie régionale. Les modèles cadavériques pour l'anesthésie régionale ont principalement été mis au point afin d'améliorer la visualisation des structures anatomiques et de l'aiguille. ${ }^{20}$ Parmi les nombreux modèles, les cadavres embaumés selon la technique de Thiel sont réputés fournir les meilleures propriétés physiques et fonctionnelles pour les blocs des nerfs périphériques ${ }^{21,22}$ et devraient donc peut-être être validés pour évaluer les blocs de plans interfasciaux.

Il convient également de déterminer la composition idéale du produit injecté pour évaluer la dispersion du produit après une technique d'analgésie régionale. Les produits injectés utilisés pour évaluer la dispersion cadavérique sont variés tant dans leur composition que par leur consistance, et comprennent le bleu de méthylène ${ }^{9-11}$ et le latex, ${ }^{8}$ lesquels ont tous deux été utilisés dans des études de blocs ESP; des formulations plus complexes ont également été utilisées pour d'autres blocs de plans interfasciaux. ${ }^{23,24}$ La viscosité variable de ces solutions pourrait avoir un impact sur la dispersion qui en résulte, et les colorants aqueux pourraient mieux se disperser à travers les tissus que les colorants de consistance plus visqueuse.

Indépendamment de la composition du produit injecté, la technique pour réaliser ces blocs doit elle aussi être standardisée. En effet, la direction de l'aiguille/du biseau et la pression générée pendant l'injection pourraient également avoir un impact sur la dynamique liquidienne et, ainsi, sur la dispersion résultante du colorant. ${ }^{25} \mathrm{La}$ description détaillée de la technique d'injection constitue une information particulièrement importante afin de comprendre et de déterminer la dispersion du produit injecté qui en résulte; il convient d'en tenir compte lors de l'interprétation des résultats d'études cadavériques. Une évaluation cohérente doit prendre en compte toutes les considérations techniques spécifiques, comme par exemple de savoir si l'injection a été faite à l'aide d'une approche où l'aiguille est visualisée dans le plan ou non, ou d'une approche médiale à latérale vs latérale à médiale. Dans le cas des blocs interfasciaux tels qu'un ESP, une autre considération telle que l'injection via une aiguille en direction céphalique ou caudale pourrait également avoir un impact important sur la dispersion du produit injecté. En outre, il est tout aussi important de savoir si la personne réalisant le bloc possède une expérience adéquate avec la technique lorsque des interventions plus récentes sont évaluées, étant donné que l'expérience pourrait elle aussi influencer la réussite du bloc.

Même si l'on parvenait à standardiser certaines étapes des études cadavériques en anesthésie régionale, ces études pourraient malgré tout ne pas expliquer complètement le mécanisme de l'analgésie, les techniques d'anesthésie régionale pouvant être influencées tant par les effets 
locorégionaux que systémiques des anesthésiques locaux. ${ }^{26,27}$ En ce qui a trait aux effets locorégionaux, les tissus musculaires et les fascias de la zone visée par le bloc peuvent se contracter, ${ }^{28}$ et les études cadavériques ne nous permettent pas de déterminer si de tels changements dans la dynamique musculo-squelettique influencent la dispersion du produit injecté. De plus, l'effet analgésique clinique d'un anesthésique local observé lors d'une absorption systémique ne peut clairement pas être évalué par une étude cadavérique. Ceci est particulièrement important étant donné que certains anesthésiques locaux, tels que la lidocaïne, ont clairement démontré des effets analgésiques puissants lors de leur administration intraveineuse. ${ }^{29}$

Par conséquent, les études anatomiques et radiologiques ont pour objet d'expliquer la plausibilité d'une technique et ne tiennent pas compte des caractéristiques des tissus vivants, de la variabilité anatomique, de la courbe d'apprentissage de l'opérateur, ou du biais de l'observateur. Malgré les écueils de ces études anatomiques, l'important est qu'il semble y avoir une variabilité considérable dans la dispersion du produit injecté. Ceci étant dit, bien que le mécanisme de l'analgésie en soi ait besoin d'être étudié de façon plus approfondie, il est également important de réfléchir à l'utilité des blocs interfasciaux dans notre pratique quotidienne. Il est essentiel de générer des données probantes cliniques de qualité et en temps opportun pour ces techniques avant que de nouvelles techniques ne soient proposées comme norme de soins.

\section{Insuffisance de données probantes de qualité élevée pour les blocs plus récents : vaut-il la peine d'attendre?}

On considère que les études randomisées contrôlées (ERC) multicentriques (ainsi que les méta-analyses de ces dernières) génèrent les données probantes de la plus haute qualité concernant l'efficacité clinique d'un médicament, d'un dispositif ou d'une technique. Cependant, il n'existe quasiment pas d'études comparatives de qualité élevée portant sur les blocs de plans interfasciaux plus récents. Alors que les études monocentriques sont souvent critiquées pour leur manque de rigueur scientifique et de validité externe ${ }^{30}$ les études multicentriques d'envergure sont non seulement très onéreuses, mais prennent également longtemps à réaliser. La génération de données probantes en temps opportun est cruciale : en effet, tout retard dans la génération de données probantes de qualité élevée quant à l'innocuité ou à l'efficacité d'une technique avant son adoption pourrait freiner l'accès de patients éligibles à des techniques potentiellement supérieures, alors que l'adoption généralisée de ces techniques avant que des données probantes concluantes ne soient disponibles va à l'encontre des principes de bienfaisance. En outre, comme le soulignent Wallis et coll., ${ }^{17}$ dès qu'une intervention est considérée comme la norme de soins, il peut être difficile de retirer une telle technique de la pratique malgré des données probantes réfutant son utilité. La collecte et l'analyse de données à partir des registres de patients, des analyses des données massives et des études observationnelles d'envergure pourraient être nécessaires afin d'évaluer l'utilité et l'innocuité des techniques d'anesthésie régionales émergentes.

\section{Éléments à considérer lors de l'évaluation de nouvelles techniques analgésiques $<0\}$}

Lorsqu'on s'intéresse à l'efficacité analgésique, les médecins doivent examiner avec soin la conception de l'étude et les limitations associées à chaque type d'étude (voir tableau). Une évaluation systématique et étape par étape a été mise au point (tableau) pour simuler les phases de développement d'une étude clinique en médecine. ${ }^{31}$ À l'aide de cette approche, une fois les études précliniques (qui, en anesthésie régionale, équivalent aux études cadavériques) réalisées, le médicament ou la technique à l'étude passe par les diverses phases d'évaluation. Les études exploratoires sont les premières phases (phase 0 : présentations/séries de cas rapportant les résultats de l'expérience préliminaire, posologie optimale, effets néfastes ou complications d'un bloc innovant); on examine ensuite l'efficacité clinique dans des études comparatives, d'abord auprès de populations limitées (phase 2: comparable à une ERC monocentrique), puis dans des populations plus vastes (phase 3: ERC multicentriques d'envergure ou études non randomisées bien conçues). Les blocs plus récents doivent également être évalués de temps à autre à l'aide d'une évaluation des technologies de santé (ETS) établie, qui consiste en une évaluation multidisciplinaire et systématique des données probantes en ce qui touche à l'innocuité, à l'efficacité et à l'impact économique et social d'un dispositif médical, médicament ou intervention, nouveau ou existant, afin d'aider à la prise de décision. ${ }^{32}$ Nous assistons à une prise de conscience croissante de l'importance des résultats non analgésiques pour le confort postopératoire du patient, et une revue systématique récente a tenté de standardiser ces critères d'évaluation, lesquels comprennent notamment les nausées et vomissements postopératoires, des échelles concernant la qualité de la récupération, la récupération gastro-intestinale, la mobilisation et la qualité du sommeil. ${ }^{33}$ Le fait de soumettre les interventions d'anesthésie régionales existantes et nouvelles à des ETS à intervalles réguliers pourrait peut-être correspondre à une étude 
Tableau L'évaluation de l'efficacité analgésique d'une nouvelle technique d'anesthésie régionale

\begin{tabular}{|c|c|c|c|}
\hline Type d'étude & $\begin{array}{l}\text { Caractéristiques de l'étude à prendre en } \\
\text { compte }\end{array}$ & À garder à l'esprit & Niveau de données probantes \\
\hline Études cadavériques & $\begin{array}{l}\text { - Le modèle cadavérique est-il réaliste? } \\
\text { - Le produit injecté influence-t-il la } \\
\text { dispersion? } \\
\text { - Variations anatomiques? } \\
\text { - Considérations techniques : pression } \\
\text { d'injection, orientation du biseau ou } \\
\text { de l'aiguille, courbe d'apprentissage/ } \\
\text { expérience de l'opérateur }\end{array}$ & $\begin{array}{l}\text { - Les résultats sont-ils confirmés ou } \\
\text { réfutés par d'autres études? } \\
\text { - Biais de l'opérateur et de l'observateur }\end{array}$ & - Études de plausibilité \\
\hline $\begin{array}{l}\text { Série / présentations } \\
\text { de cas }\end{array}$ & $\begin{array}{l}\text { - Les indications pour le bloc pourraient } \\
\text { varier } \\
\text { - Cela pourrait dénoter une utilité } \\
\text { clinique et aider à générer une } \\
\text { hypothèse d'étude }\end{array}$ & $\begin{array}{l}\text { - Biais de publication (les études } \\
\text { négatives pourraient ne pas être } \\
\text { publiées par souci d'un manque } \\
\text { d'expérience avec la nouvelle } \\
\text { technique) }\end{array}$ & $\begin{array}{l}\text { - Données probantes anecdotiques } \\
\text { (utiles pour l'expérience } \\
\text { clinique précoce et pour la } \\
\text { communication des effets } \\
\text { néfastes) }\end{array}$ \\
\hline ERC monocentrique & $\begin{array}{l}\text { - Validité interne } \\
\text { - Validité externe }\end{array}$ & $\begin{array}{l}\text { - Il est rare de réfuter ou d'accepter les } \\
\text { résultats d'une seule ERC pour en tenir } \\
\text { compte dans la pratique }\end{array}$ & $\begin{array}{l}\text { - Études génératrices } \\
\text { d'hypothèses } \\
\text { - A besoin d'études réplicatives }\end{array}$ \\
\hline ERC multicentrique & $\begin{array}{l}\text { - La validité externe peut être } \\
\text { problématique si les critères } \\
\text { d'inclusion sont trop stricts }\end{array}$ & - Demande souvent temps et moyens & $\begin{array}{l}\text { - Données probantes définitives } \\
\text { (mais pourraient ne pas être } \\
\text { disponibles à temps) }\end{array}$ \\
\hline $\begin{array}{l}\text { Études non } \\
\text { randomisées } \\
\text { (études } \\
\text { observationnelles, } \\
\text { études des registres, } \\
\text { études des bases de } \\
\text { données) }\end{array}$ & $\begin{array}{l}\text { - Pourraient manquer de validité interne } \\
\text { - Peuvent constituer une approche plus } \\
\text { pragmatique }\end{array}$ & $\begin{array}{l}\text { - Pourrait nécessiter des méthodologies } \\
\text { adaptées afin d'imiter une étude } \\
\text { clinique }\end{array}$ & $\begin{array}{l}\text { - Pourraient constituer la } \\
\text { meilleure forme de données } \\
\text { probantes en l'absence de } \\
\text { données probantes concluantes }\end{array}$ \\
\hline
\end{tabular}

$\mathrm{ERC}=$ étude randomisée contrôlée

clinique de phase 4 , dans laquelle les facteurs systémiques et les aspects non analgésiques du confort du patient seraient également pris en compte.

Les praticiens individuels et les institutions doivent examiner les meilleures données probantes disponibles pour chacune des techniques. $<0\}$ Selon nous, il faut au minimum que des ERC monocentriques soient réalisées avant qu'une technique ne soit adoptée, et cette technique doit être réévaluée à intervalles réguliers, lorsque des données probantes de plus haut niveau sont mises à jour. L'impact à long terme d'une technique analgésique plus récente devrait tenir compte de son efficacité analgésique ainsi que d'autres facteurs tels que ses effets secondaires (nausées/vomissements), les résultats autres que la douleur (temps nécessaire jusqu'à reprise de l'ambulation ou durée du séjour hospitalier), sa facilité de réalisation (particulièrement par des anesthésiologistes ne pratiquant pas régulièrement l'anesthésie régionale), son profil d'innocuité, sa facilité de maintien/suivi, sa rentabilité et d'autres aspects d'ordre organisationnel (voir figure). ${ }^{34} \mathrm{Le}$ recours à un système de gradation pragmatique, comme le propose Mudumbai et coll. ${ }^{34}$ constitue un pas dans la bonne direction, étant donné qu'il tient compte d'autres facettes des soins tels que l'amélioration des résultats cliniques, l'augmentation de l'accès aux soins, la réduction des disparités et l'amélioration de l'efficacité.

Bien que nous soyons conscients que les médecins qui ont hâte d'offrir de nouvelles interventions ont à cœur l'intérêt de leurs patients, les données d'efficacité et d'innocuité de ces blocs doivent constamment être mises à jour, équilibrant ainsi les risques et les avantages dans notre pratique quotidienne.

Conflicts of interest None declared.

Editorial responsibility This submission was handled by Dr. Hilary P. Grocott, Editor-in-Chief, Canadian Journal of Anesthesia.

\section{Conflit d'intérêt Aucun.}

Responsabilité éditoriale Cet article a été traité par Dr Hilary P. Grocott, rédacteur en chef, Journal canadien d'anesthésie.

\section{References}

1. Bos EM, Schut ME, de Quelerij M, Kalkman CJ, Hollmann MW, Lirk $P$. Trends in practice and safety measures of epidural 
analgesia: report of a national survey. Acta Anaesthesiol Scand 2018; 62: 1466-72.

2. Wong J, Lim SS. Epidural analgesia in a paediatric teaching hospital: trends, developments, and a brief review of literature. Proceedings of Singapore Healthcare 2018; 27: 49-54.

3. Pirrera B, Alagna $V$, Lucchi $A$, et al. Transversus abdominis plane (TAP) block versus thoracic epidural analgesia (TEA) in laparoscopic colon surgery in the ERAS program. Surg Endosc 2018; 32: 376-82.

4. Elsharkawy H, Pawa A, Mariano ER. Interfascial plane blocks: back to basics. Reg Anesth Pain Med 2018; 43: 341-6.

5. Aponte A, Sala-Blanch X, Prats-Galino A, Masdeu J, Moreno LA, Serneus LA. Anatomical evaluation of the extent of spread in the erector spinae plane block: a cadaveric study. Can J Anesth 2019; 66: this issue. DOI: https://doi.org/10.1007/s12630-019-01399-4

6. Forero M, Rajarathinam $M$, Adhikary SD, Chin KJ. Erector spinae plane block for the management of chronic shoulder pain: a case report. Can J Anesth 2018; 65: 288-93.

7. Costache I, Pawa A, Abdallah FW. Paravertebral by proxy - time to redefine the paravertebral block. Anaesthesia 2018; 73: 1185-8.

8. Yang HM, Choi YJ, Kwon HJ, O J, Cho TH, Kim SH. Comparison of injectate spread and nerve involvement between retrolaminar and erector spinae plane blocks in the thoracic region: a cadaveric study. Anaesthesia 2018; 73: 1244-50.

9. Adhikary SD, Bernard S, Lopez H, Chin KJ. Erector spinae plane block versus retrolaminar block: a magnetic resonance imaging and anatomical study. Reg Anesth Pain Med 2018; 43: 756-62.

10. Vidal E, Giménez H, Forero M, Fajardo M. Erector spinae plane block: a cadaver study to determine its mechanism of action (Spanish). Rev Esp Anestesiol Reanim 2018; 65: 514-9.

11. Ivanusic J, Konishi $Y$, Barrington MJ. A cadaveric study investigating the mechanism of action of erector spinae blockade. Reg Anesth Pain Med 2018; 43: 567-71.

12. Rafi AN. Abdominal field block: a new approach via the lumbar triangle. Anaesthesia 2001; 56: 1024-6.

13. Tsai HC, Yoshida T, Chuang TY, et al. Transversus abdominis plane block: an updated review of anatomy and techniques. Biomed Res Int 2017; 2017: 8284363.

14. Ma N, Duncan JK, Scarfe AJ, Schuhmann S, Cameron AL. Clinical safety and effectiveness of transversus abdominis plane (TAP) block in post-operative analgesia: a systematic review and meta-analysis. J Anesth 2017; 31: 432-52.

15. Colibaseanu DT, Osagiede O, Merchea A, et al. Randomized clinical trial of liposomal bupivacaine transverse abdominis plane block versus intrathecal analgesia in colorectal surgery. Br J Surg 2019. DOI: https://doi.org/10.1002/bjs.11141.

16. Baeriswyl $M$, Zeiter $F$, Piubellini D, Kirkham KR, Albrecht E. The analgesic efficacy of transverse abdominis plane block versus epidural analgesia: a systematic review with meta-analysis. Medicine (Baltimore) 2018; 97: e11261.

17. Wallis CJ, Detsky AS, Fan E. Establishing the effectiveness of procedural interventions: the limited role of randomized trials. JAMA 2018. DOI: https://doi.org/10.1001/jama.2018.16329.

18. University of Oxford. Oxford Centre for Evidence-based Medicine. Levels of Evidence (March 2009). Available from URL: https://www.cebm.net/2009/06/oxford-centre-evidence- based-medicine-levels-evidence-march-2009/ (accessed April 2019).

19. Eisma R, Wilkinson T. From, "silent teachers" to models. PLoS Biol 2014; 12: e1001971.

20. Kessler J, Moriggl B, Grau T. Ultrasound-guided regional anesthesia: learning with an optimized cadaver model. Surg Radiol Anat 2014; 36: 383-92.

21. Benkhadra M, Faust A, Ladoire $S$, et al. Comparison of fresh and Thiel's embalmed cadavers according to the suitability for ultrasound-guided regional anesthesia of the cervical region. Surg Radiol Anat 2009; 31: 531-5.

22. Munirama S, Eisma R, Columb M, Corner GA, McLeod GA. Physical properties and functional alignment of soft-embalmed Thiel human cadaver when used as a simulator for ultrasoundguided regional anaesthesia. Br J Anaesth 2016; 116: 699-707.

23. Sondekoppam $R V$, Ip V, Johnston DF, et al. Ultrasound-guided lateral-medial transmuscular quadratus lumborum block for analgesia following anterior iliac crest bone graft harvesting: a clinical and anatomical study. Can J Anesth 2018; 65: 178-87.

24. Dam M, Moriggl B, Hansen CK, Hoermann R, Bendtsen TF, Børglum $J$. The pathway of injectate spread with the transmuscular quadratus lumborum block: a cadaver study. Anesth Analg 2017; 125: 303-12.

25. Greenhalgh $K$, Womack $J$, Marcangelo $S$. Injectate spread in erector spinae plane block. Anaesthesia 2019; 74: 126-7.

26. Mokini Z, Vitale $G$. Blocks of the anterior abdominal wall: local and systemic effect? Anesth Analg 2015; 120: 1428.

27. Groudine $S$. Transversus abdominis plane blocks and systemic absorption. Anesth Analg 2014; 119: 1002.

28. Schleip $R$, Gabbiani $G$, Wilke $J$, et al. Fascia is able to actively contract and may thereby influence musculoskeletal dynamics: a histochemical and mechanographic investigation. Front Physiol 2019; 10: 336.

29. Beaussier M, Delbos A, Maurice-Szamburski A, Ecoffey C, Mercadal L. Perioperative use of intravenous lidocaine. Drugs 2018; 78: 1229-46.

30. Bellomo R, Warrillow SJ, Reade MC. Why we should be wary of single-center trials. Crit Care Med 2009; 37: 3114-9.

31. Canadian Cancer Society. Types and phases of clinical trials. Available from URL: https://www.cancer.ca/en/cancerinformation/diagnosis-and-treatment/clinical-trials/types-andphases-of-clinical-trials/?region=qc (accessed April 2019).

32. Lampe K, Mäkelä M, Garrido M, et al. The HTA core model: a novel method for producing and reporting health technology assessments. Int J Technol Assess Health Care 2009. DOI: https:// doi.org/10.1017/s0266462309990638.

33. Myles PS, Boney $O$, Botti $M$, et al. Systematic review and consensus definitions for the Standardised Endpoints in Perioperative Medicine (StEP) initiative: patient comfort. Br J Anaesth 2018; 120: 705-11.

34. Mudumbai SC, Auyong DB, Memtsoudis SG, Mariano ER. A pragmatic approach to evaluating new techniques in regional anesthesia and acute pain medicine. Pain Manag 2018; 8: 475-85.

Publisher's Note Springer Nature remains neutral with regard to jurisdictional claims in published maps and institutional affiliations. 